\title{
Case report of delayed radiotherapy-related pleural effusion following chest radiotherapy for lymphoma
}

\author{
Panxiao Shen ${ }^{1}$, Yunxiang Zeng ${ }^{1}$, Weizhan Luo ${ }^{1}$, Yingying Gu${ }^{2}$, Jinlin Wang ${ }^{1}$ \\ ${ }^{1}$ Department of Respiratory Disease, ${ }^{2}$ Department of Pathology, the State Key Laboratory of Respiratory Disease, China Clinical Research Centre \\ for Respiratory Disease, Guangzhou Institute of Respiratory Health, Guangzhou 510120, China \\ Correspondence to: Jinlin Wang. The State Key Laboratory of Respiratory Disease, China Clinical Research Centre for Respiratory Disease, \\ Guangzhou Institute of Respiratory Health, First Affiliated Hospital of Guangzhou Medical University, 151 Yanjiang Rd, Guangzhou 510120, China. \\ Email: drjlwang@126.com.
}

Submitted Apr 08, 2018. Accepted for publication Jul 24, 2018.

doi: $10.21037 /$ jtd.2018.07.113

View this article at: http://dx.doi.org/10.21037/jtd.2018.07.113

\section{Introduction}

Radiotherapy is one of the commonly used methods for the management of lymphoma and solid tumors and is known to be associated with various complications that usually occur in a considerable proportion of treated patients (1). Post-radiotherapy complications are typically classified into acute, subacute, and chronic. Delayed radiotherapyrelated pleural effusion (DRPE), although recognized as a chronic complication, has been scarcely reported in the literature, probably due to a low occurrence rate among the relevant population. DRPE is usually atypically manifested in clinical symptoms and signs, with a greatly varied interval period from the radiation exposure to its development (2-7). The poorly-defined characteristics, the unavailability of data, such as the exact incidence of DRPE, and the absence of clearly identified diagnostic criteria pose a great challenge for clinicians to make a conclusive diagnosis of DRPE. DRPE's occurrence may be more common than clinicians think, and it is frequently misdiagnosed; thus, it can potentially develop for up to several years after radiation exposure against a complicated background of multiple comorbidities $(3,6,7)$. Such condition needs to be considered for disease management.

In the current study, DRPE occurred in a 42-year-old woman, 25 years after undergoing radiotherapy for cervical lymphoma. This case is very rare in view of such a long interval and may therefore contribute to an improved understanding of DRPE and its accurate diagnosis and timely treatment.

\section{Case presentation}

A 42-year-old woman with exertional dyspnea for 6 months was admitted to our institution for recurrent right pleural effusion (Figure 1) of unknown etiology. She denied a history of cough, fever, chest pain, hemoptysis, night sweats, fatigue, itching, or weight loss. Empirical antibiotics were administered following multiple thoracocenteses, but the pleural effusion remained accumulated. Her past medical history was notable for lymphoma that was diagnosed 25 years ago and managed by radiotherapy to the supraclavicular fossae and mediastinum and six cycles of chemotherapy, among other treatments. Histopathological classification data and treatment details were unavailable. The treatment was successful, and no evidence suggesting a recurrence of lymphoma was observed during regular follow-ups.

The patient was well-nourished without a history of heart disease, liver disease, or kidney disease. Physical examination revealed radiotherapy-induced pachulosis, pigmentation, and erythematous patches over the neck and upper chest without observed cervical or supraclavicular jugular venous distention. Pulmonary examination was remarkable for decreased breath sounds with dullness to percussion on the right chest. Laboratory findings, including routine blood test, liver function panel, thyroid function test, C-reactive protein, and erythrocyte sedimentation rate suggested no abnormality. Sputum smears and cultures for fungi, acidfast bacilli, and other bacteria were negative. Antinuclear antibody, rheumatoid factor antibody, antineutrophilic 


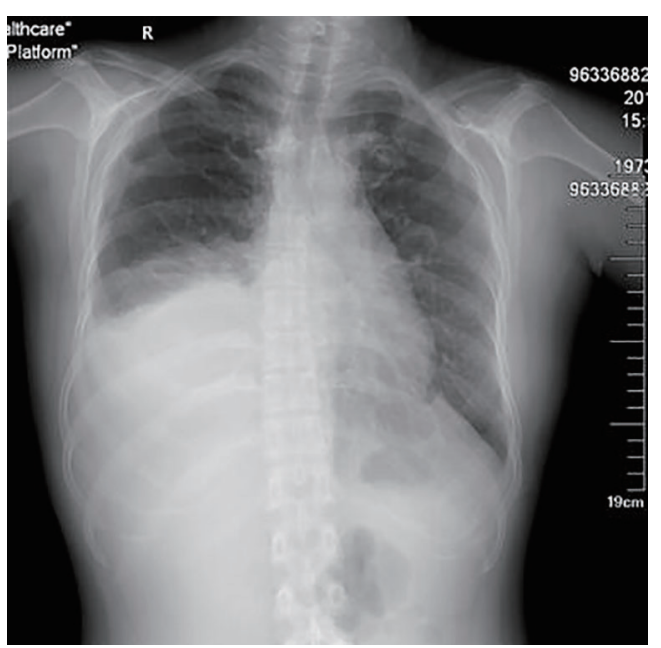

Figure 1 Chest X-ray reveals mild tracheal cartilage calcification.
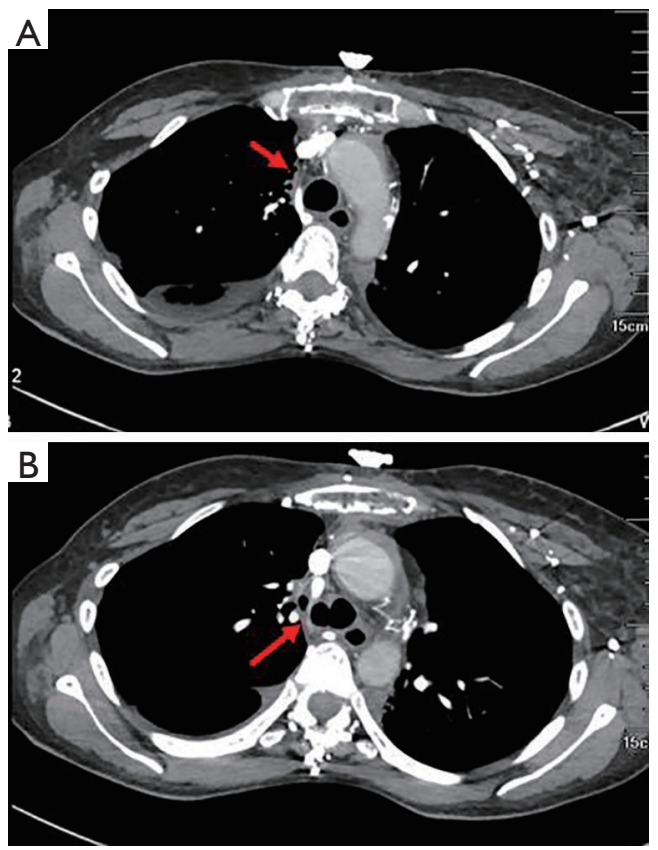

Figure 2 Chest HRCT demonstrates right pleural effusion and mediastinal fibrosis. (A) Fibrosis on the right side of trachea (arrow); (B) fibrosis in the right hilum and stenosis in right main bronchia (arrow). HRCT, high-resolution computed tomography.

cytoplasmic antibody, and anti-cyclic citrullinated peptide antibody were not detected.

Chest high-resolution computed tomography (HRCT) (Figure 2) demonstrated right pleural effusion and enlarged lymph nodes in bilateral armpits without significant

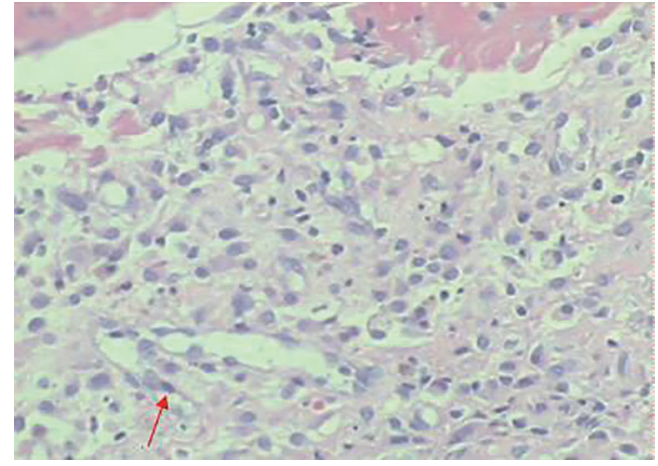

Figure 3 Histopathological examination shows multiple reactive mesothelial cells (arrow). No neoplastic cells are observed (HE staining and the magnification of microscope is missing).

parenchymal abnormalities. Notable findings, such as mediastinal mass, airway anomalies, pleural nodularity, or pulmonary emboli, were not detected. There was a slight fibrosis in the mediastinal lymph nodes. Pericardial effusion was excluded by transthoracic echocardiography, by which relevant measurements were also obtained, including a left ventricular ejection fraction of $71 \%$ with a normal chamber size and an estimated right ventricular systolic pressure of $40 \mathrm{mmHg}$.

Thoracocentesis revealed a transudative nature of the pleural effusion, with a fluid protein level of $3.07 \mathrm{~g} / \mathrm{dL}$ and a lactate dehydrogenase level of $63.0 \mathrm{U} / \mathrm{L}$. Lung tumor markers, such as carcino-embryonic antigen, carbohydrate antigen-125 ( $\left.\mathrm{CA}_{125}\right), \mathrm{CA}_{153}$, and $\mathrm{CA}_{199}$, were negative. Pleural fluid bacterial and Mycobacterium tuberculosis cultures and acid-fast bacilli smears were also negative. Biochemical tests for fluid adenosine deaminase levels (2.7 U/L) and NT pro-brain natriuretic peptide also produced normal results. Pleural biopsy indicated multiple reactive mesothelial cells but no neoplastic cells (Figure 3). Left axillary lymph node biopsy revealed inflammatory reactive hyperplasia with no evidence supporting a diagnosis of lymphoma.

On the basis of the radiotherapy history, clinical manifestations, and pleural pathological analyses, a diagnosis of DRPE was considered for our patient. Prednisolone at a daily dose of $30 \mathrm{mg}$ was administered as part of the treatment, and the patient responded with significantly subsided pleural effusion and a gradually improved physical condition. The dosage of prednisolone was titrated down as symptoms improved until the patient was discharged from our hospital. 
An 8-month follow-up showed a satisfactory recovery of the patient, with a repeated HRCT demonstrating a small amount of residual pleural effusion in the right costophrenic sulcus.

\section{Discussion}

DRPE secondary to radiation treatment for lymphoma has rarely been described in the literature. Rodríguez-García et al. (5) and Fragoulis et al. (6) respectively reported a case involving recurrent pleural effusion 8 and 23 years following mediastinal radiotherapy for Hodgkin's disease. Pericarditis, a frequently observed post-radiotherapy complication, was also detected in both cases. Chotirmall et al. (3) presented DRPE occurrence in a patient with non-Hodgkin's lymphoma 36 years after radiotherapy. For this case, making a conclusive diagnosis of DRPE was challenging because only $1 \mathrm{~L}$ of pleural fluid was drained from the thoracic cavity. Malignant diseases, including non-Hodgkin's lymphoma recurrence, could not be excluded due to absence of pleural biopsy and reliable pathological information. Furthermore, missing follow-up data diminished evidence for DRPE. Therefore, the case described in the current study is the first confirmed diagnosis of DRPE with a long interval between the radiation treatment and its onset. DRPE in this case was clearly differentiated from other confounding conditions, such as pericarditis.

Considering that the published studies associated with DRPE are mostly delineated individual cases, identifying the exact incidence and defining the characteristics of DRPE is greatly challenging (5-7). DRPE may vary greatly from normal to extremely abnormal in clinical manifestation and biochemical indicators, such as the cell classification and count, $\mathrm{pH}$, glucose, and protein content of pleural effusion $(2,3,5,6)$. Thoracotomy or pleural biopsy usually suggests chronic nonspecific pleural inflammation and multiple reactive mesothelial hyperplasia $(2,4,5)$. In the current case, massive unilateral pleural effusion in the right chest, which was identified as transudative in nature based on Light's criteria, repeatedly occurred 25 years after radiotherapy. However, the manifestations, laboratory tests, imaging, and pathologic findings of our patient were non-specific and did not suggest any signs of infection or malignancy, thereby making it difficult to perform an accurate diagnosis.

A definitive diagnosis of DRPE is always presumptive. It necessitates a thorough work-up to rule out other disorders $(8,9)$. Considering the history of the patient, the possibility of persistent pleural effusion related to lymphoma or a secondary malignancy must be strongly considered first $(3,10,11)$. However, laboratory or imaging findings failed to provide any convincing evidence for our patient to support such a diagnosis. Another suspicion that should be raised from our physicians was malignant pleural mesothelioma (MPM), which has been noted as a secondary malignancy in patients who received radiotherapy for Hodgkin's disease even if associated with asbestos exposure (12-15). In the present study, the possibility of MPM was ruled out through a comprehensive evaluation and long-term follow up. Cardiovascular-related pleural effusion must also be considered because mediastinal radiotherapy may cause delayed cardiovascular complications (16). In the current case, pleural effusion induced by cardiogenic disorders was ruled out by the clinical manifestations and electrocardiogram and echocardiogram results, which suggested the absence of cardiovascular abnormalities. In addition, we have tried to find out more details of radiotherapy, but unfortunately, details of radiotherapy including dose and cycles could not be obtained because the interval is such a long time as 25 years and the patient could not memorize it precisely. So far, there is no a clear diagnostic criterion or guideline for such entity due to its rarity. According to previous literature, details of radiotherapy are not necessary for diagnosis, but other disorders causing PE must be excluded. Thus, the diagnosis of DRPE was eventually established for our patient after a number of other possibilities were eliminated in the differential diagnosis.

Currently, interpreting the mechanism of DRPE is established mainly on the hypothesis deduced from individual cases and is believed to be more complicated than we thought. The complexity may be responsible for the enormous variation in the interval ranging from 2 to 23 years (2-7). Constrictive pericarditis with or without tamponade, superior vena cava obstruction, lymphatic obstruction, and the complications of mediastinal fibrosis may be involved in the development and progress of DRPE $(4,5)$. In the current study, constrictive pericarditis and superior vena cava obstruction were excluded through clinical and imaging analysis. Thus, we suspected the occurrence of impaired lymphatic drainage resulting from mediastinal fibrosis and chronic pleuritic, which were induced by radiation therapy; these conditions led to recurrent pleural effusion. However, we noticed that fibrosis was also seen on left side (Figure 2), left-sided pleural effusion did not occur. This discrepancy probably could be explained by different severity of damage to both 
sides. In general, pleural effusion that is not secondary to radiation pleuritis tends to occur at $>1$ year following the radiation treatment (5). In the current case, reactive mesothelium hyperplasia as revealed by pleural biopsy probably suggests chronic radiation pleuritis, which may be implicated in the pathogenesis of DRPE. In addition, the reactive mesothelial cells in the pleural biopsy is the key point to exclude MPM. However, the presence of any correlation between the development of pleural effusion and the radiation quantity, modality of the radiotherapy, or post-radiotherapy management is unclear. Further investigation is therefore necessary to identify the related factors to achieve an optimal mitigation or amelioration for this late complication.

DRPE treatment is still being determined. Pleurodesis $(2,4)$, a procedure that aims to achieve adhesion between visceral and parietal pleura and obliterate the pleural space using sclerosing agents, is considered as an option to prevent pleural effusion recurrence. Fragoulis et al. (6) reported improvement after treatment with oral doxycycline for a case of delayed effusive pericarditis and recurrent pleural effusion after radiation treatment for Hodgkin's lymphoma. However, the mechanism and concentration of doxycycline in the pleural fluid after oral administration are unclear. The use of non-steroidal anti-inflammatory drugs, such as indomethacin, has been reported in such cases (5). For our patient, significant regression of recurrent pleural effusion was observed following thoracic drainage and corticosteroid treatment. Long-term follow-up was performed as routine management.

In conclusion, DRPE must be considered for differential diagnosis in patients with recurrent pleural effusion even many years after radiotherapy. Increasing DRPE awareness will contribute to an accurate diagnosis. Comprehensive work-ups are essential to further explore the mechanism and treatment of this disease.

\section{Acknowledgements}

None.

\section{Footnote}

Conflicts of Interest: The authors have no conflicts of interest to declare.

Informed Consent: Written informed consent was obtained from the patient for publication of this manuscript and any accompanying images.

\section{References}

1. Hull MC, Morris CG, Pepine CJ, et al. Valvular dysfunction and carotid, subclavian, and coronary artery disease in survivors of hodgkin lymphoma treated with radiation therapy. JAMA 2003;290:2831-7.

2. Morrone N, Gama e Silva Volpe VL, et al. Bilateral pleural effusion due to mediastinal fibrosis induced by radiotherapy. Chest 1993;104:1276-8.

3. Chotirmall SH, Low TB, Murphy P, et al. Delayed radiotherapy-related effusions: malignant or not malignant, that is the question. Respirology 2008;13:754; author reply 755 .

4. Whitcomb ME, Schwarz MI. Pleural effusion complicating intensive mediastinal radiation therapy. Am Rev Respir Dis 1971;103:100-7.

5. Rodríguez-García JL, Fraile G, Moreno MA, et al. Recurrent massive pleural effusion as a late complication of radiotherapy in Hodgkin's disease. Chest 1991;100:1165-6.

6. Fragoulis KN, Handrinou E, Papadopoulos V, et al. Delayed effusive pericarditis and recurrent pleural effusion after radiation treatment for Hodgkin's disease responsive to per os doxycycline. Eur J Haematol 2006;76:176-9.

7. Kumar R, Patel G, Kichenadasse G, et al. Delayed onset of benign pleural effusion following concurrent chemoradiotherapy for inoperable non-small-cell lung cancer. Intern Med J 2015;45:218-21.

8. Maskell N, British Thoracic Society Pleural Disease Guideline Group. British Thoracic Society Pleural Disease Guidelines--2010 update. Thorax 2010;65:667-9.

9. McGrath EE, Anderson PB. Diagnosis of pleural effusion: a systematic approach. Am J Crit Care 2011;20:119-27; quiz 128.

10. Bernardeschi P, Bonechi I, Urbano U. Recurrent pleural effusion as manifesting feature of primitive chest wall Hodgkin's disease. Chest 1988;94:424-6.

11. Schaapveld M, Aleman BM, van Eggermond AM, et al. Second Cancer Risk Up to 40 Years after Treatment for Hodgkin's Lymphoma. N Engl J Med 2015;373:2499-511.

12. Lerman $Y$, Learman $Y$, Schachter $P$, et al. Radiation associated malignant pleural mesothelioma. Thorax 1991;46:463-4.

13. Neugut AI, Ahsan H, Antman KH. Incidence of malignant pleural mesothelioma after thoracic radiotherapy. Cancer 
1997;80:948-50.

14. Kramer G, Gans S, Rijnders A, et al. Long-term survival of a patient with malignant pleural mesothelioma as a late complication of radiotherapy for Hodgkin's disease treated with 90yttrium-silicate. Lung Cancer 2000;27:205-8.

Cite this article as: Shen P, Zeng Y, Luo W, Gu Y, Wang J. Case report of delayed radiotherapy-related pleural effusion following chest radiotherapy for lymphoma. J Thorac Dis 2018;10(8):E625-E629. doi: 10.21037/jtd.2018.07.113
15. Cavazza A, Travis LB, Travis WD, et al. Post-irradiation malignant mesothelioma. Cancer 1996;77:1379-85.

16. Adams MJ, Lipsitz SR, Colan SD, et al. Cardiovascular status in long-term survivors of Hodgkin's disease treated with chest radiotherapy. J Clin Oncol 2004;22:3139-48. 